\title{
Guest Editorial \\ Computational Solutions to Large-Scale Data Management and Analysis in Translational and Personalized Medicine
}

M EDICINE is undergoing a revolution that is transforming the nature of healthcare from reactive to preventive. The changes are catalyzed by a new systems approach to disease that focuses on integrated diagnosis, treatment, and prevention of disease in individuals. This is expected to replace our current mode of medicine over the coming years with a personalized and predictive treatment. While the goal is clear, the path is fraught with challenges.

Our success in addressing these challenges depends on our ability to properly interpret the large-scale, high-dimensional datasets that are generated by modern technologies, manage dimensionality problems, and extract meaningful prognostic/diagnostic biomarkers, since it is becoming increasingly clear that a comprehensive analysis of biological systems requires the integration of all fingerprints of cellular function: genome sequence, maps of gene expression, protein expression, metabolic output, and in vivo enzymatic expression (activity). This need for integration is especially clear in the case of complex, multifactorial diseases, such as cancer.

At the same time, the Virtual Physiological Human (VPH) framework aims to define models and technologies that once fully established will enable the investigation of the human body as a whole, eventually leading to a better healthcare system that offers personalized care solutions, more holistic approach to medicine and a preventative approach to the treatment of disease. Although consensus exists about what the fundamental tools are (integration of high-throughput data from several biologic scales, high-definition imaging, and computational modeling), no such consensus exists as to what are the most promising scientific approaches in responding to these challenges.

A growing awareness is found that, despite significant technological advances in various domains of relevance, fundamental obstacles separate systems biology from clinical applications. Applying engineering precepts to biological systems has spawn the field of systems biology to investigate a network of interacting components, including the coordination of internal systems of living organisms such as endocrine, nervous, and respiratory with gene and gene product expression, and behavior and environmental factors, and understand how these components together contribute to the disease initiation and progression,

Digital Object Identifier 10.1109/JBHI.2014.2315513 biological development, and health. Proceeding from systems biology, systems medicine incorporates complex and dynamic biochemical, physiological, and environmental interactions between all components of disease and health that sustain living organisms. For example, the caring of the whole person exemplifies the integration and correlation at multiple scales of biology, expanding medicine beyond reductionism. Bridging these gaps will require a focused and concerted effort in addressing various questions, such as:

1) What defines systems medicine as a discipline?

2) What should systems medicine seek to accomplish?

3) How to develop optimal frameworks for large-scale data sharing, distributed scientific collaboration, and collective research efforts?

4) How to exploit data from hospital information systems, Electronic Health Records (EHRs) and Personalized Health Records (PHRs) and how to curate these data?

5) How should knowledge from multifaceted, disparate data sources be collected and assembled into ontologies relevant to the education and practice of systems medicine?

6) How do we develop and evaluate appropriate algorithms for biomarker selection, diagnosis and prognosis as well as therapeutic agent identification and evaluation?

7) How do we manage the problems of high dimensionality of large scale medical data?

8) How are multiscale data to be represented and/or synthesized by corresponding multiscale models?

9) How should models be represented, stored, managed, and shared?

10) Health recommender systems: How to deliver personalized health information to the care givers and patients effectively?

11) What is the burden of proof that such models are valid and predictive of clinically relevant outcomes?

Furthermore, because of the necessary multiscale nature of the models, bridging embedded levels of organization from molecules, organelles, cells, tissues, organs, and all the way to individuals, environmental factors, populations, and ecosystems, systems medicine aims to discover and select the key factors at each level and integrate them into models of translational relevance, which include measurable readouts and clinical predictions. 
The current Special Issue includes a selected number of papers presented at the 12th IEEE International Conference on BioInformatics and BioEngineering (BIBE 2012), Nov. 11-13, 2012, Cyprus (http://bibe2012.cs.ucy.ac.cy/) under a special session with the same theme, in addition to papers submitted following an open call for papers.

The Special Issue presents experiences as well as technological and scientific developments stemming from some flagship projects funded by the EU under the FP7 framework programme aiming to bring together researchers working in the fields of infrastructures and technologies for integrative biomedical research, ICT for predictive and translational medicine and the VPH community at large.

A total of 15 papers are included under the following scientific subdomains: 1) mHealth, Wearable Systems and Telemonitoring Services (five papers), 2) Medical Imaging (four papers), and 3) Computational Biology (six papers).

It is expected that technological advances in the areas covered in this Special Issue promise great precision in diagnosis, individualization of patient care, opportunity for earlier intervention, further development of information infrastructure of translational and personalized medicine for the delivery of more cost-effective and individualized healthcare services that would also facilitate their deployment worldwide for the benefit of all patients.
MANOLIS TSIKNAKIS, Guest Editor

TEI Crete and FORTH, 71004 Heraklion, Greece (tsiknaki@ics.forth.gr).

VASILIS J. PROMPONAS, Guest Editor

University of Cyprus, Nicosia 1678, Cyprus

(vprobon@ucy.ac.cy).

NORBERT GRAF, Guest Editor

University of the Saarland, 66123 Saarbrücken, Germany (graf@uks.eu).

MAY D. WANG, Guest Editor

Georgia Institute of Technology and Emory University

Atlanta, GA 30332 USA

(maywang@bme.gatech.edu).

STEPHEN T. C. WONG, Guest Editor

Houston Methodist Research Institute, Weill Cornell

Medical College, Houston, TX 77030

(stwong@tmhs.org).

NikOLAOS BourbaKIS, Guest Editor

Wright State University, Dayton, OH 45435 USA

(nikolaos.bourbakis@wright.edu).

Constantinos S. PATTICHIS, Guest Editor

University of Cyprus, Nicosia 1678, Cyprus

(pattichi@ucy.ac.cy). 